\title{
Zeros at Infinity for Affine Nonlinear Control Systems
}

\author{
HENK NIJMEIJER, MEMBER, IEEE, AND JOHANNES M. SCHUMACHER, MEMBER, IEEE
}

\begin{abstract}
A definition of zeros at infinity for affine nonlinear control systems is proposed. The definition is local, which means that we exclude certain singularities. We argue the reasonableness of our definition by showing its relevance to the problem of nonlinear decoupling. In particular, we give a necessary and sufficient condition for the solvability of the general regular decoupling problem for affine systems in terms of the zeros at infinity.
\end{abstract}

\section{INTRODUCTION}

$\mathbf{T}$ HE purpose of the present paper is to study the decoupling problem and its connection to zeros at infinity for the class of affine nonlinear systems. The connection between the two subjects has been well established in the context of linear systems (cf. [1], [2]), and it turns out that it is possible to establish quite similar results for nonlinear systems-as long as one restricts oneself, as we do in this paper, to a "local" point of view, i.e., one allows the introduction of assumptions that will hold on open parts of the state manifold but possibly not on the entire manifold as such. Our main result (Theorem 4.1) gives a necessary and sufficient condition for the solvability of the regular static-state feedback noninteracting control problem for affine systems (the problem is defined in Section IV). It is shown in Theorem 3.1 how this necessary and sufficient condition can be interpreted in terms of zeros at infinity. The decouping results of the present work extend those of [24], where the treatment was restricted to situations in which the number of scalar inputs equals the number of vector outputs. Of course, the development sketched above would not be possible without having available a definition of "zeros at infinity" for the class of affine systems. For more restricted classes of nonlinear systems, indexes which could serve to define zeros at infinity have been introduced by Hirschorn [6] and Isidori [8]. We consider it a point of major interest of the present paper that here, for the first time, the notion of "zeros at infinity" is defined for the full class of affine systems. It is shown in [26] that our definition encompasses those given by Hirschorn and Isidori.

It is perhaps worthwhile to expand on what the concept of "zeros at infinity" means (see also [3] for linear systems, [23] for nonlinear systems). Basically, the zeros at infinity are numbers that indicate the orders of integration in a (multivariable) system. Consider first a linear single-input single-output system $\dot{x}=A x$ $+b u, y=c^{T} x$. The "order of integration" in such a system can be defined, for instance, as the lowest number $k$ for which the input function $u$ appears explicitly in the expression for the $k$ th derivative of $y$. Since $\dot{y}=c^{T} A x+c^{T} b u, \ddot{y}=c^{T} A^{2} x+c^{T} A b u$ $+c^{T} b \dot{u}$, etc., it is clear that this order of integration could also be expressed algebraically as the lowest value of $k$ for which the number $c^{T} A^{k-1} b$ is unequal to zero.

Because the development around infinity of the transfer function $g(s)=c^{T}(s I-A)^{-1} b$ is $g(s)=c^{T} b s^{-1}+c^{T} A b s^{2}+$ $\cdots$, yet another way of expressing the order on integration would

Manuscript received October 17, 1983; revised October 15, 1984. Paper recommended by Associate Editor, J. Baillieul.

H. Nijmeijer is with the Department of Applied Mathematics, Twente University of Technology, Enschede, The Netherlands.

J. M. Schumacher was with the Econometric Institute, Erasmus University, Rotterdam, The Netherlands. He is now with the Centre for Mathematics and Computer Science, Amsterdam, The Netherlands. be that it is the unique value of $k$ for which $s^{k} g(s)$ has a finite and nonzero value at infinity. Following the standard terminology of function theory, this number is also called the order of the zero at infinity of $g(s)$. Note that the first definition that we gave for "order of integration" would also apply to nonlinear systems. The situation is more complicated if we turn to multivariable systems. For decoupled scalar systems (with a diagonal transfer matrix), it is clear that the proper definition of the zeros at infinity for the system as a whole would be to take the zeros at infinity of each channel separately. In general, however, one has to reorganize the input- and output-channels in such a way that the integration structure is displayed by a set of numbers. In the linear case, this can be done by using the concept of a "bicausal matrix," i.e., a proper rational matrix which also has a proper rational inverse, so that it has, in this sense, neither poles nor zeros at infinity. The idea is that multiplication of a transfer matrix by a bicausal matrix does not "essentially" change the integration structure. One then proves (see [3], [14]) that for every strictly proper rational matrix $G(s)$ there exist bicausal matrices $B_{1}(s)$ and $B_{2}(s)$ such that

$$
\begin{gathered}
B_{1}(s) G(s) B_{2}(s)=\left[\begin{array}{cc}
\Delta(s) & 0 \\
0 & 0
\end{array}\right] \\
\Delta(s)=\operatorname{diag}\left(s^{-d_{1}}, \cdots, s^{-d_{r}}\right) .
\end{gathered}
$$

Moreover, the numbers $d_{1}, \cdots, d_{r}$ are determined uniquely by $G(s)$. It is then natural to call these numbers the (orders of the) zeros at infinity of the system described by $G(s)$.

The above definition is not easily extended to nonlinear systems since it is given in terms of the transfer matrix. Fortunately, there are also characterizations available directly in state-space terms. Such a characterization was already given in [14], but a recent and slightly different version due to Malabre [13] turns out to be more useful for our purposes. Let a system $\Sigma(A, B, C)$ be given, with state-space $X$, and consider the " $V *$-algorithm" [37]

$$
\begin{gathered}
V^{0}=X \\
V^{k+1}=\left\{x \in V^{k} \mid A x \in V^{k}+\operatorname{Im} B\right\} .
\end{gathered}
$$

In a finite number of steps, this sequence of subspaces tends to a limit, which is denoted by $V^{*}$. It can then be shown that the number

$$
p^{k} \operatorname{def} \operatorname{dim}\left(\operatorname{Im} B \cap V^{k-1}\right)-\operatorname{dim}\left(\operatorname{Im} B \cap V^{*}\right)
$$

is equal to the number of zeros at infinity of order $\geq k$, as defined above. So, the zeros at infinity can be recovered from the numbers $p^{k}$ as defined by (1.5). Malabre's [13] proof of this is rather indirect; for a short proof, see [26]. It is this characterization of the integration structure that will be generalized to nonlinear systems in the next section.

\section{Differential Geometric Structure Theory}

We consider an affine nonlinear control system

$$
\dot{x}(t)=A(x(t))+\sum_{i=1}^{m} B_{i}(x(t)) u_{i}(t)
$$


where $x$ are local coordinates of a smooth $n$-dimensional manifold $M, A, B_{1}, \cdots, B_{m}$ are smooth vector fields on $M$ and $u_{i}:$ R $_{+} \rightarrow$ R is a piecewise smooth input function, $i \in \boldsymbol{m}$. Together with the dynamics (2.1), we consider the output functions

$$
z_{i}(t)=C_{i}(x(t)), \quad i \in \boldsymbol{k}
$$

where $C_{i}: M \rightarrow N_{i}$ is a smooth map from $M$ to a smooth $p_{i}$ dimensional manifold $N_{i}, p_{i} \geq 1, i \in k$. We assume that each $C_{i}$, $i \in k$, is a surjective submersion. Throughout the paper we will make the following standard assumptions for the systems (2.1), (2.2):
A1) $\operatorname{dim} \Delta_{0}:=\operatorname{dim} \operatorname{span}\left\{B_{1}, \cdots, B_{m}\right\}=m$
A2) the rank of the map $C:=\left(C_{1}, \cdots, C_{k}\right)$

$$
: M \rightarrow N_{1} \times \cdots \times N_{k} \quad \text { equals } p_{1}+\cdots+p_{k}
$$

A3) system (2.1) satisfies the strong accessibility-rank condition (see [33], [18]).

We allow here static-state feedback, i.e., an admissible control law has the form

$$
u=\alpha(x)+\beta(x) v
$$

where $\alpha: M \rightarrow R^{m}, \beta: M \rightarrow R^{m \times m}$ are smooth functions. To keep as much open-loop control as possible, we assume that $\beta(x)=$ $\left(\beta_{i j}(x)\right)_{i, j}$ is nonsingular for all $x \in M ; v=\left(v_{1}, \cdots, v_{m}\right)^{t} \in \mathbb{R}^{m}$ represents a new input. By applying the feedback law (2.6) to (2.1) we obtain as new dynamics

$$
\dot{x}(t)=\tilde{A}(x(t))+\sum_{i=1}^{m} \tilde{B}_{i}(x(t)) v_{i}(t)
$$

where

$$
\begin{gathered}
\tilde{A}(x)=A(x)+\sum_{i=1}^{m} B_{i}(x) \alpha_{i}(x), \\
\tilde{B}_{i}(x)=\sum_{j=1}^{m} B_{j}(x) \beta_{j i}(x) .
\end{gathered}
$$

Next we come to one of the basic concepts in the "differential geometric approach" to nonlinear system theory. For detailed accounts we refer to [4], [8]-[12], [16]-[28] and to [37] for the linear counterpart.

Definition 2.1: A fixed-dimensional involutive distribution $D$ on $M$ is locally controlled invariant if, locally around each point $x_{0} \in M$ there exists a control law (2.6) such that the modified dynamics (2.7) satisfies

$$
\begin{gathered}
{[\tilde{A}, D] \subset D,} \\
{\left[\tilde{B}_{i}, D\right] \subset D, \quad i \in m .}
\end{gathered}
$$

There also exists a definition of global controlled invariance [8], [9], but the advantage of the local concept above the global one is that the following test is available to determine whether or not a distribution is locally controlled invariant.

Theorem 2.2: Let $D$ be an involutive distribution on $M$ of fixed dimension and assume that $D \cap \Delta_{0}$ has fixed dimension. Then, $D$ is locally controlled invariant if and only if

$$
\begin{gathered}
{[A, D] \subset D+\Delta_{0},} \\
{\left[B_{i}, D\right] \subset D+\Delta_{0}, \quad i \in m .}
\end{gathered}
$$

An important class of controlled invariant distributions is given by the following.
Definition 2.3: A fixed-dimensional involutive distribution $D$ on $M$ is a regular controllability distribution if, locally around each point $x_{0} \in M$ there exists a control law (2.6) such that

$$
\begin{gathered}
{[\tilde{A}, D] \subset D,} \\
{\left[\tilde{B}_{i}, D\right] \subset D, \quad i \in m}
\end{gathered}
$$

and

$$
\begin{aligned}
& D=\text { involutive closure of } \\
& \left.\qquad a d_{\tilde{A}^{\prime}}^{k} \Delta_{0} \cap D, a d_{\bar{B}_{i}}^{k} \Delta_{0} \cap D \mid k \in \mathbb{Z}_{+}, i \in \boldsymbol{m}\right\} .
\end{aligned}
$$

Or equivalently (see [18]) $D=$ involutive closure of $\left\{a d_{\tilde{A}}^{k} \tilde{B}_{j}\right.$, $\operatorname{ad}_{\tilde{B}_{i}}^{k} \tilde{B_{j}} \mid k \in \mathbb{Z}_{+}, i \in \boldsymbol{m}$ and $\left.j \in I \subset \boldsymbol{m}\right\}$, for a certain subset $I \subset$

As in the linear geometric theory (see [37]) locally controlled invariant distributions and regular local controllability distributions play an important role in the (local) solution of synthesis problems like the disturbance decoupling problem and the noninteracting control problem (see [4], [8]-[12], [16]-[28]). In this context one is especially interested in supremal elements satisfying Definition 2.2 or (2.5), which are contained in a given fixed-dimensional involutive distribution $K$ on $M$. However, in general, these supremal elements may not exist. In order to overcome this problem we consider the following algorithm:

$$
\left\{\begin{array}{l}
V^{0}=T M \\
V^{\mu+1}=K \cap \Delta^{-1}\left(\Delta_{0}+V^{\mu}\right)
\end{array}\right.
$$

where

$$
\Delta^{-1}(V)=\{X \in V(M) \mid[\Delta, X] \subset V\}
$$

and $\Delta$ is the affine distribution associated with (2.1)

$$
\Delta(x)=A(x)+\Delta_{0}(x) .
$$

It is straightforward to show that the algorithm (2.12) converges in at most $\operatorname{dim} K$ steps to a limit, which will be denoted as $V_{k}^{*}$, so $V^{*}=V^{\operatorname{dim} K}$.

Now, in general, the (involutive) distributions $V^{\mu}, \mu \geq 0$, appearing in (2.12) will not have constant dimension. However, for analytic systems the $V^{\mu}{ }^{\prime} s, \mu \geqslant 0$, are of constant dimension on an open and dense submanifold $M^{\prime}$ of $M$. Now, if we exclude all possible singularities in the dimensions of the $V^{\mu} s, \mu \geq 0$ and $V^{\mu} \cap \Delta_{0}, \mu \geq 0$, then we know (see, e.g., [4], [9], [16]) that $V_{K}^{*}$ is the maximal element in the family of all controlled invariant distributions contained in $K$. Therefore, we will make the following basic assumption (valid on open parts of $M$ ).

Assumption 2.4: For each $\mu \geq 0$, the distributions $V^{\mu}$ and $V^{\mu}$ $\cap \Delta_{0}$ will have fixed dimension, where $V^{\mu}$ is defined in (3.12).

The (nonlinear) algorithm (2.12) contains structural information about a control system, as shown in [37] for the linear case. In what follows we will mimic the linear theory on infinite zeros as far as possible. For linear references see, e.g., [35] and [26]. Consider a smooth nonlinear control system (2.1) together with one output function $C$ as in (2.2). By assumption, the function $C$ being a surjective submersion-induces a fixed-dimensional involutive distribution $\operatorname{Ker} C_{*}$, on $M$. Therefore, we may apply algorithm (2.12) to $\operatorname{Ker} C_{*}$, and assume that Assumption 2.4 holds in the case. Then supremal locally controlled invariant distribution contained in $\operatorname{Ker} C_{*}$ is denoted as $V^{*}$ and satisfies $V^{*}$ $=V^{k+1}=V^{k}$ for all $k \geq n-p$ where $p=\operatorname{rank} C$. Now we define a set of integers by the following.

Definition 2.5:

$$
p^{\mu}:=d\left(\Delta_{0} \cap V^{\mu-1}\right)-d\left(\Delta_{0} \cap V^{*}\right), \quad \mu>0 .
$$

Associated with the sequence $\left\{p^{\mu}\right\}_{\mu=1}^{n-p}$ we define another list by the following. 


\section{Definition 2.6:}

$n^{\mu}:=$ number of $p^{\nu}$ s which are greater than or equal to $\mu$.

There is a one-to-one correspondence between the sequences $\left\{p^{\mu}\right\}_{\mu=1}^{n-p}$ and $\left\{n^{\mu}\right\}_{\mu=1}^{p^{1}}$ given by $(2.16)$ and $p^{\mu}=$ number of $n^{\nu}$ s which are greater than or equal to $\mu$.

As in the linear case (see [13], [26]) we will say that the nonlinear system (2.1), with output

$$
z=C(x)
$$

has $p^{1}$ zeros at infinity of orders $\left\{n^{\mu}\right\}$. As we have seen in Section I, these integers play an important role in the linear theory (as, for example, in Silverman's structure algorithm), but also in the solution of the noninteracting control problem; see [1], [2]. In the next sections it will be shown that in the general nonlinear noninteracting control problem, the integers $\left\{p^{\mu}\right\}$ (or $\left\{n^{\mu}\right\}$ ) play the same role as in the linear theory of [1], [2]. It is for this reason that we have chosen to call the $n^{\mu}$ 's the orders of the $p^{1}$ infinite zeros. Further explanation is given in [26].

Remark: For general nonlinear systems of the form (locally)

$$
\left\{\begin{array}{l}
\dot{x}=f(x, u) \\
y=h(x, u)
\end{array}\right.
$$

one can define zeros at infinity in the following way. We form an "extended system" (cf. [27]) by introducing a new input function $v$ as follows:

$$
\dot{u}=v \text {. }
$$

The extended system (2.19), (2.20) with $\left(\begin{array}{l}x \\ u\end{array}\right)$ as state and $v$ as input is affine, and we can apply the above definition (see also [28]). From the orders of zero at infinity so obtained, we subtract one in order to compensate for the integration we have added. Note that, in this way, one may find zeros at infinity of order zero; this is in agreement with the linear situation. Of course, one has to show that this definition is consistent in the sense that if (2.19) happens to be affine, then the definition given above agrees with the direct definition given earlier. This has been done in [25]. In the rest of this paper, we will limit ourselves to affine systems.

Let us finally say a few words on controllability distributions. Again it can be shown (see [12], [18]) that there exists a supremal regular local controllability distribution $R_{K}^{*}$, contained in a given fixed-dimensional involutive distribution $K$ on $M$. Notice, however, that $R_{K}^{*}$ is not necessarily of constant dimension. As in the linear theory there is no direct algorithm for computing $R_{K}^{*}$. The easiest way of computing $R_{K}^{*}$ is with the aid of $V_{K}^{*}$. This can be summarized in the following procedure.

Step 1: Compute $V_{R}^{*}$ (assume $V_{K}^{*}$ has constant dimension).

Step 2: Compute appropriate $\tilde{A}, \widetilde{B}_{1}, \cdots, \widetilde{B}_{m}$ which leave $V_{K}^{*}$ invariant.

Step 3: Compute $\Delta_{0} \cap V_{K}^{*}$.

Step 4: $R$ 类 $=$ involutive closure of $\left\{a d_{\tilde{A}}^{k} \Delta_{0} \cap V_{K}^{*}, a d_{B_{B}}^{k} \Delta_{0} \cap\right.$ $\left.V_{K}^{*} \mid k \in Z_{+}, i \in \boldsymbol{m}\right\}$.

Notice that, almost by construction, the following identity holds (cf. [18]):

$$
\Delta_{0} \cap R_{K}^{*}=\Delta_{0} \cap V_{K}^{*}
$$

which will be used in the sequel.

\section{STRUCTURE AT INFINITY FOR MULTIPLE OUtPuts}

We now consider the system (2.1), (2.2) under the standard assumptions (2.3)-(2.5). While (2.4) holds, we have that for each $I \subset \boldsymbol{k}$ the involutive distribution $\bigcap_{j \in I} \operatorname{Ker} C_{j *}$ is of constant dimension, and therefore we may apply the algorithm (2.12) for each of them. Assuming that Assumption 2.4 holds for each sequence of distributions, we obtain the corresponding supremal local controlled invariant elements. We will list them as follows:

$V^{*}=$ supremal locally controlled invariant distribution in Ker $C_{*}$.

$V_{I}^{*}=$ supremal locally controlled invariant distribution in

$$
\bigcap_{j \in I} \operatorname{Ker} C_{j *}, \quad I \subset k \text {. }
$$

We also write

$$
D_{I}^{*}=V_{k}^{*} \backslash I, \quad I \subset k
$$

and

$$
R_{I}^{*=} \text { supremal regular local controllability distribution in }
$$

The corresponding lists of orders of the zeros at infinity will be denoted as follows:

$$
\begin{gathered}
p_{i}^{\mu}=d\left(\Delta_{0} \cap V_{i}^{\mu-1}\right)-d\left(\Delta_{0} \cap V_{i}^{*}\right), i \in k, \mu>0 \\
p^{\mu}=d\left(\Delta_{0} \cap V^{\mu-1}\right)-d\left(\Delta_{0} \cap V^{*}\right), \mu>0 \\
q_{i}^{\mu}=d\left(\Delta_{0} \cap D_{i}^{\mu-1}\right)-d\left(\Delta_{0} \cap D_{i}^{*}\right), i \in k, \mu>0 \\
p^{\mu}(I)=d\left(\Delta_{0} \cap V_{I}^{\mu-1}\right)-d\left(\Delta_{0} \cap V_{I}^{*}\right), I \subset k, \mu>0 \\
q^{\mu}(I)=d\left(\Delta_{0} \cap D_{I}^{\mu-1}\right)-d\left(\Delta_{0} \cap D_{I}^{*}\right), I \subset k, \mu>0
\end{gathered}
$$

It is convenient in this notation that we set

$$
V_{b}^{*}=T M \text { and } D_{b}^{*}=V^{*} \text {. }
$$

The following relations are immediate:

$$
V_{I}^{\mu}=D_{k \backslash I}^{\mu}, \quad I \subset k, \mu>0 .
$$

If $I \subset J \subset \boldsymbol{k}$, we have

$$
\begin{array}{ll}
D_{I}^{\mu} \subset D_{J}^{\mu}, & \mu>0, \\
V_{I}^{\mu} \supset V_{J}^{\mu}, & \mu>0, \\
V^{\mu} \subset D_{i}^{\mu} \subset V_{j}^{\mu}, & \mu>0, i \neq j, i, j \in k .
\end{array}
$$

Furthermore, we note that by definition (3.2)

$$
p_{i}^{\mu}=p^{\mu}(\{i\}), q_{i}^{\mu}=q^{\mu}(\{i\}), \quad i \in k, \mu>0
$$

and

$$
q^{\mu}(I)=p^{\mu}(\boldsymbol{k} \backslash I), \quad I \subset k, \mu>0 .
$$

For what follows we need one other definition. A function $\phi: P(k)$ $\rightarrow Z_{+}$is called a weight function if

1) $\phi(\emptyset)=0$,

2) for all $I, J \in P(\boldsymbol{k})(=$ the family of subsets of $\boldsymbol{k})$

we have $\phi(I \cup J)=\phi(I)+\phi(J)-\phi(I \cap J)$.

After these preliminaries we come to the main theme of this section. Consider the indentity

$$
\Delta_{0}=\sum_{i \in k} \Delta_{0} \cap D_{i}^{*}
$$

Relation (3.7), to which we will refer as the noninteraction condition (this terminology will be fully justified in Section IV), 
is equivalent to certain relations among the indexes $p_{i}^{\mu}, p^{\mu}$, and $p^{\mu}(\cdot)$. Notice that for linear systems it is known that (3.7) is equivalent to, cf. [1]

$$
p^{\mu}=\sum_{i \in k} p_{i}^{\mu}, \quad \text { for all } \mu>0
$$

Here we will give an extension of this result.

Theorem 3.1: Assume that for all $I \subset k, V_{I}^{*}$ satisfies Assumption 2.4. Then the following are equivalent:

a) $\Delta_{0}=\sum_{i \in k} \Delta_{0} \cap D_{i}^{*}$

b) $p^{\mu}=\sum_{i \in k} p_{i}^{\mu}$, for all $\mu>0$,

c) $p^{\mu}: p(k) \rightarrow Z_{+}$is a weight function, for all $\mu>0$.

For the proof of this theorem we need some preliminary results.

Lemma 3.2: Suppose Assumption 2.4 holds for all $D *, I \subset \boldsymbol{k}$. Then, if for certain $\mu \geq 0$

$$
D_{I \cap J}^{\mu}=D_{I}^{\mu} \cap D_{J}^{\mu}
$$

and

$$
\Delta_{0}=\Delta_{0} \cap D_{I}^{\mu}+\Delta_{0} \cap D_{J}^{\mu}
$$

then also

$$
D_{I \cap J}^{\mu+1}=D_{I}^{u+1} \cap D_{I}^{u+1}
$$

Proof:

$$
\begin{aligned}
D_{I}^{\mu+1} \cap D_{J}^{\mu+1} & =\bigcap_{j \in k \backslash I} \operatorname{Ker} C_{j^{*}} \cap \bigcap_{j \in k \backslash J} \operatorname{Ker} C_{j^{*}} \\
& \cap \Delta^{-1}\left[\left(\Delta_{0}+D_{I}^{\mu} \cap \cap\left(\Delta_{0}+D_{J}^{\mu}\right)\right]\right. \\
& =\bigcap_{j \in k \backslash I \cap J} \operatorname{Ker} C_{j}^{*} \\
& \cap \Delta^{-1}\left[\Delta_{0} \cap D_{I}^{\mu}+\Delta_{0} \cap D_{J}^{\mu}+D_{I}^{\mu} \cap D_{J}^{\mu}\right] \\
& =\bigcap_{j \in k \backslash I \cap J} \operatorname{Ker} C_{j}^{*} \cap \Delta^{-1}\left[\Delta_{0}+D_{I \cap J}^{\mu}\right] \\
& =D_{I \cap J}^{\mu+1} .
\end{aligned}
$$

Lemma 3.3: Suppose Assumption 2.4 holds for all $D_{l}^{*}, I \subset \boldsymbol{k}$. Then,

$$
\Delta_{0}=\sum_{i \in k} \Delta_{0} \cap D_{i}^{*}
$$

implies that for all $I, J \subset k, \mu \geq 0$

$$
D_{I}^{\mu} \cap D_{J}^{\mu}=D_{I \cap J}^{\mu}
$$

Proof: Choose $I, J \subset \boldsymbol{k}$ and let us first assume that $I \cup J=$ $\boldsymbol{k}$. By applying (3.4a) we have

$$
\begin{aligned}
& \Delta_{0} \supset \Delta_{0} \cap D_{I}^{*}+\Delta_{0} \cap D^{*} \supset \Delta_{0} \cap \sum_{i \in I} D_{i}^{*} \\
& +\Delta_{0} \cap \sum_{i \in J} D_{i}^{*} \supset \sum_{i \in k} \Delta_{0} \cap D_{i}^{*}
\end{aligned}
$$

So, by (3.7)

$$
\Delta_{0}=\Delta_{0} \cap D_{I}^{*}+\Delta_{0} \cap D^{*}
$$

and thus, for all $\mu \geq 0$,

$$
\Delta_{0}=\Delta_{0} \cap D_{I}^{u}+\Delta_{0} \cap D_{J}^{\mu}
$$

Induction and Lemma 3.2 lead to the desired result (3.9). Now for arbitrary $I, J \subset k$ we have

$$
D_{I}^{\mu} \cap D_{J}^{\mu} \subset D_{I}^{\mu} \cap D_{J \cup k \backslash I}^{\mu}
$$

and because $I \cup J \cup k \backslash I=k$, we have that

$$
D_{I}^{\mu} \cap D_{J \cup k \backslash I}^{\mu}=D_{I \cap(J \cup k \backslash D)}^{\mu}=D_{I \cap \sigma^{\circ}}^{\mu}
$$

On the other hand

$$
D_{I \cap J}^{\mu} \subset D_{I}^{\mu} \cap D_{J}^{\mu}
$$

so by (3.12), (3.13), and (3.14) we obtain

$$
D_{I \cap J}^{\mu}=D_{I}^{\mu} \cap D_{J}^{\mu}
$$

Lemma 3.4: Suppose Assumption 2.4 holds for all $D^{*}, I \subset k$. Then, for all $\mu \geq 0$,

$$
\Delta_{0}=\sum_{i \in k} \Delta_{0} \cap D_{i}^{\mu}
$$

if and only if

$$
\forall I, J \subset k \Delta_{0} \cap D_{I}^{\mu}+\Delta_{0} \cap D_{J}^{\mu}=\Delta_{0} \cap D_{I \cup J}^{\mu} .
$$

Proof:

$\left(\Leftrightarrow \Delta_{0}=\Delta_{0} \cap D_{k}^{*}=\Delta_{0} \cap D_{k}^{\mu}=\Delta_{0} \cap D_{1}^{\mu}+\Delta_{0} \cap D_{\{2, \cdots, k\}}^{\mu}\right.$

$$
=\sum_{i \in k} \Delta_{0} \cap D_{i}^{\mu}
$$

( $\Rightarrow$ ) Let $I, J \subset k$. Then for all $\mu \geq 0$,

$$
\begin{aligned}
\Delta_{0} \cap D_{I \cup J}^{\mu} & =\left(\Delta_{0} \cap D_{I}^{\mu}+\Delta_{0} \cap D_{J}^{\mu}+\Delta_{0} \cap D_{k \backslash I \cup J}^{\mu}\right) \cap D_{I \cup J}^{\mu} \\
& =\Delta_{0} \cap D_{I}^{\mu}+\Delta_{0} \cap D_{J}^{\mu}+\Delta_{0} \cap D_{I \cup J}^{\mu} \cap D_{k \backslash I \cup J}^{\mu} \\
& =\Delta_{0} \cap D_{I}^{\mu}+\Delta_{0} \cap D_{J}^{\mu}+\Delta_{0} \cap D_{\emptyset}^{\mu}(\text { by Lemma 3.3) } \\
& =\Delta_{0} \cap D_{I}^{\mu}+\Delta_{0} \cap D_{J}^{\mu}+\Delta_{0} \cap V^{\mu} \\
& =\Delta_{0} \cap D_{I}^{\mu}+\Delta_{0} \cap D_{J}^{\mu}
\end{aligned}
$$

We are now able to prove the main theorem of this section.

Proof (of Theorem 3.1):

$(a \Rightarrow c)$ We have by Lemmas 3.3 and 3.4, for all $I, J \subset k$ and $\mu$ $\geq 0$, that

$$
\Delta_{0} \cap D_{I}^{\mu}+\Delta_{0} \cap D_{J}^{\mu}=\Delta_{0} \cap D_{I \cup J}^{\mu}
$$

$$
\left(\Delta_{0} \cap D_{I}^{\mu}\right) \cap\left(\Delta_{0} \cap D_{J}^{\mu}\right)=\Delta_{0} \cap D_{I \cap J}^{\mu}
$$

and so by (3.3) it follows for all $I, J \subset k, \mu \geq 0$ that

$$
\begin{gathered}
\Delta_{0} \cap V_{I}^{\mu}+\Delta_{0} \cap V_{J}^{\mu}=\Delta_{0} \cap V_{I \cap J}^{\mu}, \\
\left(\Delta_{0} \cap V_{I}^{\mu}\right) \cap\left(\Delta_{0} \cap V_{J}^{\mu}\right)=\Delta_{0} \cup V_{I \cup J}^{\mu} .
\end{gathered}
$$

Therefore, for all $\mu>0$

$$
\begin{gathered}
p^{\mu}(\emptyset)=d\left(\Delta_{0} \cap V_{\emptyset}^{\mu-1}\right)-d\left(\Delta_{0} \cap V_{\emptyset}^{*}\right)=m-m=0 \\
p^{\mu}(I \cup J)=d\left(\Delta_{0} \cap V_{I \cup J}^{\mu-1}\right)-d\left(\Delta_{0}-V_{I \cup J}^{*}\right) .
\end{gathered}
$$


Using (3.17) we have

$$
\begin{aligned}
d\left(\Delta_{0} \cap V_{I U J}^{\mu-1}\right)= & d\left[\left(\Delta_{0} \cap V_{I}^{\mu-1}\right) \cap\left(\Delta_{0} \cap V_{J}^{\mu-1}\right)\right] \\
= & d\left(\Delta_{0} \cap V_{I}^{\mu-1}\right)+d\left(\Delta_{0} \cap V_{J}^{\mu-1}\right) \\
& -d\left(\Delta_{0} \cap V_{I}^{\mu-1}+\Delta_{0} \cap V_{J}^{\mu-1}\right)
\end{aligned}
$$

and by (3.18)

$$
d\left(\Delta_{0} \cap V_{I}^{\mu-1}+\Delta_{0} \cap V_{J}^{\mu-1}\right)=d\left(\Delta_{0} \cap V_{I \cap J}^{\mu}\right)
$$

Furthermore, (3.21) and (3.22) hold true if we replace $\mu$ by *, i.e., by taking $\mu$ sufficiently large. Combination of these expressions, together with (3.20), (3.21), and (3.22) leads to

$$
\begin{aligned}
p^{\mu}(I \cup J)= & d\left(\Delta_{0} \cap V_{I}^{\mu-1}\right)+d\left(\Delta_{0} \cap V_{J}^{\mu-1}\right)-d\left(\Delta_{0} \cap V_{I \cap J}^{\mu-1}\right) \\
& -d\left(\Delta_{0} \cap V_{I}^{*}\right)-d\left(\Delta_{0} \cap V_{J}^{*}\right)+d\left(\Delta_{0} \cap l^{*}\right) \\
= & p^{\mu}(I)+p^{\mu}(J)-p^{\mu}(I \cap J) .
\end{aligned}
$$

So (3.19) and (3.23) readily yield that $p^{\mu}$ is a weight-function for all $\mu>0$.

$(c \Rightarrow b)$ For all $\mu>0$ we have

$$
\begin{aligned}
p^{\mu} & =p^{\mu}(k)=p^{\mu}(\{1\})+p^{\mu}(\{2, \cdots, k\})-p^{\mu}(\emptyset) \\
& =p_{1}^{\mu}+p^{\mu}(\{2, \cdots, k\})=p_{1}^{\mu}+\cdots=\sum_{i \in k} p_{i}^{\mu} .
\end{aligned}
$$

$(b \Rightarrow a)$ We will show by induction that if (3.8) holds, then for all $\mu \geq 0$

$$
\Delta_{0}=\sum_{i \in k} \Delta_{0} \cap D_{i}^{\mu}
$$

as well as

$$
V^{\mu}=\bigcap_{i \in k} V_{i}^{\mu}
$$

and

$$
D_{i}^{\mu}=\bigcap_{j \neq i} V_{j}^{\mu}, \quad i \in k
$$

Clearly the statement is true for $\mu=0$. Assume (3.24)-(3.26) hold for a certain $\mu>0$, then by repeated application of Lemma 3.2 (3.25) and (3.26) hold true for $\mu+1$. Furthermore, we have [see (3.4c)] for all $i \in \boldsymbol{k}$

$$
\Delta_{0}=\Delta_{0} \cap D_{i}^{\mu}+\Delta_{0} \cap V_{i}^{\mu} .
$$

Next we compute $d\left(\Delta_{0} \cap D_{i}^{\mu+1}+\Delta_{0} \cap V_{i}^{\mu+1}\right)$.

$$
\begin{aligned}
d\left(\Delta_{0} \cap D_{i}^{\mu+1}+\Delta_{0} \cap V_{i}^{\mu+1}\right) & =d\left(\Delta_{0} \cap D_{i}^{\mu+1}\right)+d\left(\Delta_{0} \cap V_{i}^{\mu+1}\right)-d\left(\Delta_{0} \cap D_{i}^{\mu+1} \cap V_{i}^{\mu+1}\right) \\
= & d\left(\bigcap_{j \neq i} \Delta_{0} \cap V_{j}^{\mu+1}\right)+d\left(\Delta_{0} \cap V_{i}^{\mu+1}\right)-d\left(\Delta_{0} \cap V^{\mu+1}\right) \\
\geq & \sum_{j \neq i} d\left(\Delta_{0} \cap V_{j}^{\mu+1}\right)-(k-2) m+d\left(\Delta_{0} \cap V_{i}^{\mu+1}\right) \\
& -d\left(\Delta_{0} \cap V^{\mu+1}\right) \\
= & \sum_{j \in k} d\left(\Delta_{0} \cap V_{j}^{\mu+1}\right)-(k-2) m-d\left(\Delta_{0} \cap V^{\mu+1}\right) .
\end{aligned}
$$

Using $p^{\mu}=\Sigma_{j \in k} p_{j}^{\mu}$, we obtain the following identities

$$
m-d\left(\Delta_{0} \cap V^{*}\right)=\sum_{j \in k}\left[m-d\left(\Delta_{0} \cap V_{j}^{*}\right)\right]
$$

so

$$
\sum_{j \in k} d\left(\Delta_{0} \cap V_{j}^{*}\right)-d\left(\Delta_{0} \cap V^{*}\right)=(k-1) m
$$

Moreover,

$d\left(\Delta_{0} \cap V^{\mu+1}\right)-d\left(\Delta_{0} \cap V^{*}\right)=\sum_{j \in k}\left[d\left(\Delta_{0} \cap V_{j}^{\mu+1}\right)-d\left(\Delta_{0} \cap V_{j}^{*}\right)\right]$

which, by (3.29), leads to

$$
\sum_{j \in k} d\left(\Delta_{0} \cap V_{j}^{\mu+1}\right)-d\left(\Delta_{0} \cap V^{\mu+1}\right)=(k-1) m .
$$

So from (3.28) and (3.31), we conclude

$$
d\left(\Delta_{0} \cap D_{i}^{\mu+1}+\Delta_{0} \cap V_{i}^{\mu+1}\right) \geq m,
$$

i.e.,

$$
\Delta_{0} \cap D_{i}^{\mu+1}+\Delta_{0} \cap V_{i}^{\mu+1}=\Delta_{0} .
$$

Having established (3.32) for all $i \in k$, we see

$$
\begin{aligned}
\Delta_{0} & =\bigcap_{i \in k}\left[\Delta_{0} \cap D_{i}^{\mu+1}+\Delta_{0} \cap V_{i}^{\mu+1}\right] \\
& =\sum_{i \in k} \Delta_{0} \cap D_{i}^{\mu+1}+\Delta_{0} \cap V^{\mu+1}
\end{aligned}
$$

so

$$
\Delta_{0}=\sum_{i \in k} \Delta_{0} \cap D_{i}^{\mu+1}
$$

Therefore, (3.24) is established for all $\mu \geq 0$ and (3.7) readily follows by taking $\mu$ sufficiently large.

While the numbers $p^{\mu}$ and $p_{i}^{\mu}(i \in k)$ are in one-to-one correspondence to the orders of the infinite zeros (see Definition 2.6 ), condition (3.8) can also be established by using them. Let

$n^{\mu}=$ number of $p^{\mu}$ 's which are greater than or equal to $\mu$,

$n_{i}^{\mu}=$ number of $p_{i}^{\mu}$ 's which are greater than or equal to $\mu, i \in \boldsymbol{k}$.

Then we have the following.

Corollary 3.5: Assume that for all $I \subset k, V^{*}$ satisfies Assumption 2.4. Then

$$
\Delta_{0}=\sum_{i \in k} \Delta_{0} \cap D_{i}^{*}
$$

is equivalent to

$$
\left\{n^{\mu}\right\}_{1}^{p^{1}}=\bigcup_{i \in k}^{\cdot}\left\{n^{\mu}\right\}_{1}^{p_{i}^{1}}
$$

where $U$ denotes the set theoretic union (with repeated common elements).

Remarks: i) In case the number of scalar inputs $(=m)$ equals the number of vector outputs $(=k)$, the noninteracting condition 
(3.7) reduces to a direct sum

$$
\Delta_{0}=\bigoplus_{i \in k} \Delta_{0} \cap D_{i}^{*}
$$

and

$$
\Delta_{0} \cap V^{*}=0
$$

(see [24] for details). ii) As already noted in (2.21) we can replace (3.7) by

$$
\Delta_{0}=\sum_{i \in k} \Delta_{0} \cap R_{i}^{*}
$$

which will be the starting point of the next section.

\section{The General Noninteracting Control Problem}

We now come to the generalization of the linear regular blockdecoupling problem (here regular means that one uses full control in the decoupling state feedback): see $[15,38,1]$. Let us briefly outline the input-output decoupling problem under consideration. For a more complete discussion of this topic, we refer to [24], where the same problem has been solved in case the number of scalar $V$ inputs equals the number of vector outputs. Consider the system (2.1), (2.2) under the assumptions (2.3)-(2.5). Suppose that, after applying a feedback law (2.6) the new input $v_{i}$ does not affect the output $z_{j}, j, i \in k, j \neq i$, and moreover the input $\boldsymbol{v}_{i}$ "controls" the output $z_{i}, i \in \boldsymbol{k}$. Here $\left(v_{1}, \cdots, v_{k}\right)^{t}=\left(v_{1}, \cdots\right.$, $\left.v_{m}\right)^{t}$, but some $v_{i}, i \in m$, may appear in various vector inputs $v_{j}, \dot{j}$ $\in \boldsymbol{k}$. That is, there is a partitioning

$$
m=I_{1} U \cdots U I_{k}
$$

with the property that $j \in I_{l} \Leftrightarrow v_{j}$ belongs to $v_{l}, l \in k$.

Clearly, if $v_{i} \in v_{\alpha} \cap v_{\beta}$ for some $\alpha \neq \beta \in k$, then neither $z_{j}, j$ $\neq \alpha$, nor $z_{j}, j \neq \beta$, is affected by $v_{i}$; so all outputs $z_{j}, j \in \boldsymbol{k}$, are independent of the input $v_{i}$. Therefore, excluding overlappings in the various input vectors $v_{i}, i \in \boldsymbol{k}$ leads to a partitioning $v=\left(v^{0}\right.$, $\left.v^{1}, \cdots, v^{k}\right)^{t}$ such that $\boldsymbol{v}^{0}$ does not affect $z_{j}, j \in \boldsymbol{k}$ and $\boldsymbol{v}^{i}$ does not affect $z_{j}, j \neq i$, and "controls" $z_{i}$. This allows us to rewrite the partitioning (4.1) as

$$
m=I^{0} \oplus I^{1} \oplus \cdots \oplus I^{k}
$$

with the property $j \in I^{l} \Leftrightarrow v_{j} \in v^{l}, l=0,1, \cdots, k$.

Consider the regular (local) controllability distributions

$$
\begin{aligned}
& R_{j}=\operatorname{span} \text { (involutive closure of }\left\{a d_{\tilde{A}}^{k} \widetilde{B_{i}}, a d_{\tilde{B} l}^{k} \widetilde{B_{i}} \mid\right. \\
& \left.\quad k \in \mathcal{Z}_{+}, i \in I_{j}, l \in m\right\} \text { ), } \quad j \in k .
\end{aligned}
$$

The noninteraction conditions can be nicely expressed by means of the distributions $R_{1}, \cdots, R_{k}$, namely the input $v_{i}$ does not affect $z_{j}, j \neq i$, if and only if

$$
R_{j} \subset \bigcap_{i \neq j} \operatorname{Ker} C_{i} *, \quad j \in k
$$

while $\boldsymbol{v}_{i}$ " controls" $z_{i}, i \in \boldsymbol{k}$ is equivalent to (see [20], [22], [24] for the definition of output controllability)

$$
R_{j}+\operatorname{Ker} C_{j} * T M, \quad j \in k
$$

or equivalently

$$
C_{j} *\left(R_{j}\right)=T N_{j}, \quad j \in k .
$$

The static-state feedback noninteracting control problem can now be formulated as follows.

Given the system (2.1), (2.2) find, if possible, a feedback law (2.6) such that (4.4) and (4.5) hold for the distributions defined by (4.3). This problem will be solved here in a local fashion. Given an arbitrary initial point $x_{0} \in M$ we are interested in finding a local feedback law (2.6), i.e., $\alpha$ and $\beta$ are possibly only well-defined in a neighborhood of $x_{0}$ (compare to Definition 2.1 and Theorem 2.2 on local controlled invariance).

Without any further requirements we cannot get global solutions of the above problem. The solution of the nonlinear noninteracting control problem is similar to the linear (geometric) version of this problem (see [15], [38]) so the differential geometric approach again provides a good framework for such a synthesis problem. Recall the definition (3.1d) of $R_{Y}^{*} I \subset \boldsymbol{k}$. The theorem we are after is as follows.

Theorem 4.1: Consider the system (2.1), (2.2) and assume that for all $I \subset k, V_{I}^{\mu}$ and $\mathrm{V}_{I}^{\mu} \cap \Delta_{0}$ all have fixed dimension. Then the static-state feedback noninteracting control problem is locally solvable around each point $x_{0} \in M$ if and only if

$$
\Delta_{0}=\sum_{i \in k} \Delta_{0} \cap D_{i}^{*}
$$

Furthermore, if these conditions hold, then $\left\{R_{i}^{*}\right\}_{i=1}^{k}$ is the only solution satisfying (4.4) and (4.5).

We will prove this theorem by using the following result of [24].

Theorem 4.2: Consider the system (2.1), (2.2) and assume that for all $I \subset k V_{I}^{\mu}$ and $V_{I}^{\mu} \cap \Delta_{0}, \mu>0$ all have fixed dimension. Then the static-state feedback noninteracting control problem is locally solvable around each point $x_{0} \in M$ if

$$
\Delta_{0}=\bigoplus_{i \in k} \Delta_{0} \cap D_{i}^{*}
$$

Remark: The sufficient condition (4.7), which is equivalent to $\Delta_{0}=\oplus_{i \in k} \Delta_{0} \cap R_{\mathrm{i}}^{*}$, implies that the $R_{i}^{*}, i=1, \cdots, i$ are "simultaneously integrable," that is, for each subset $I \subset k$ the distribution $\Sigma_{i \in I} R_{i}^{*}$ is involutive. This is the basic observation of [24] needed for the construction of a decoupling feedback law.

The idea to use Theorem 4.2 for proving the sufficient part of Theorem 4.1 is that we first "factor out" the maximal unobservable distribution in $\operatorname{Ker} d C$, i.e., $V^{*}$ [see (3.1a)], and then we show that the reduced system on the quotient manifold $M\left(\bmod V^{*}\right)$, exactly satisfies the sufficient condition (4.7). Note that the quotient system will have $m \backslash I^{0}$ inputs [see (4.2)]. In formalizing this we need the following results.

Lemma 4.3: If (3.7) holds, then

$$
\Delta_{0} / \Delta_{0} \cap V^{*}=\sum_{i \in k}\left(\Delta_{0} \cap D_{i}^{*}\right) /\left(\Delta_{0} \cap V^{*}\right) .
$$

Proof: By definition, we have $V^{*} \subset D_{i}^{*}$ for $i=1, \cdots, k$. Therefore,

$$
\begin{aligned}
\Delta_{0} / \Delta_{0} \cap V^{*} & =\left(\sum_{i \in k} \Delta_{0} \cap D_{i}^{*}\right) / \Delta_{0} \cap V^{*} \\
& =\sum_{i \in k}\left(\Delta_{0} \cap D_{i}^{*}\right) /\left(\Delta_{0} \cap V^{*}\right) .
\end{aligned}
$$

Lemma 4.4: If

$$
\Delta_{0} \cap V^{*}=0
$$

then (3.7) is equivalent to

$$
\Delta_{0}=\bigoplus_{i \in k} \Delta_{0} \cap D_{i}^{*}
$$

that is the distributions $\left\{\Delta_{0} \cap D_{i}^{*}\right\}_{i=1}^{k}$ are independent.

Proof: As a result of the previous section we know that (3.7) is equivalent to $p^{\mu}: p(k) \rightarrow \mathbb{Z}_{+}$being a weight function for all $\mu>$ 
0 . Therefore,

$$
\begin{aligned}
& p^{1}(k \backslash\{1\})+p^{1}(k \backslash\{2\})+\cdots+p^{2}(\boldsymbol{k} \backslash\{k\}) \\
&= p^{1}(\boldsymbol{k})+p^{1}(\boldsymbol{k} \backslash\{1,2\})+p^{1}(\boldsymbol{k} \backslash\{3\})+\cdots+p^{1}(\boldsymbol{k} \backslash\{k\}) \\
&= p^{1}(k)+p^{1}(k)+p^{1}(k \backslash\{1,2,3\})+p^{1}(k \backslash\{4\}) \\
&+\cdots+p^{1}(k \backslash\{k\})=\cdots \\
&=(k-1) p^{1}(\boldsymbol{k})+p^{1}(\emptyset)=(k-1)(m-0)=(k-1) m .
\end{aligned}
$$

So $m-d\left(\Delta_{0} \cap D_{1}^{*}\right)+m-d\left(\Delta_{0} \cap D_{2}^{*}\right)+\cdots+m-d\left(\Delta_{0}\right.$ $\left.\cap D_{k}^{*}\right)=(k-1) m$ from which we deduce

$$
d\left(\Delta_{0} \cap D_{1}^{*}\right)+d\left(\Delta_{0} \cap D_{2}^{*}\right)+\cdots+d\left(\Delta_{0} \cap D_{k}^{*}\right)=m .(4.10)
$$

Clearly, (4.10) is equivalent to (4.7).

Now we proceed with the proof of the main theorem.

Proof (of Theorem 4.1): For sufficiency, we assume that (3.7) or the equivalent (3.38) holds. The proof now proceeds in two steps. Let $x_{0} \in M$. Then we first construct a local feedback law

$$
u=\alpha(x)+\beta(x) \tilde{u}
$$

such that the modified dynamics leaves $V^{*}$ invariant, i.e.,

$$
\left[\tilde{A}, V^{*}\right] \subset V^{*}
$$

$$
\left[\tilde{B_{i}}, V^{*}\right] \subset V^{*}, \quad i \in \boldsymbol{m}
$$

[here $\tilde{A}$ and $\tilde{B_{i}}$ are as in (2.8a), (2.8b)]. This is possible by Theorem 2.2. Moreover, we may choose the vector fields $\tilde{B}_{1}$, $\cdots, \widetilde{B}_{m}$ [and thus the matrix $\beta(\cdot)$ ] such that $\widetilde{B}_{1}, \cdots, \tilde{B}_{l}, l=d\left(\Delta_{0}\right.$ $\left.\cap V^{*}\right)$ form a basis for $\Delta_{0} \cap V^{*}$. Choosing Frobenius coordinates on a neighborhood $0\left(x_{0}\right)$ of $x_{0}$ such that $V^{*}=$ span $\left\{\partial / \partial x_{1}\right\}, \partial / \partial x_{1}$ possibly being a vector, (4.12) amounts to

$$
\begin{gathered}
\tilde{A}\left(x_{1}, x_{2}\right)=\left(\begin{array}{c}
\tilde{A}^{1}\left(x_{1}, x_{2}\right) \\
\tilde{A}^{2}\left(x_{2}\right)
\end{array}\right), B_{i}\left(x_{1}, x_{2}\right)=\left(\begin{array}{c}
\tilde{B}_{i}^{1}\left(x_{1}, x_{2}\right) \\
0
\end{array}\right), \quad i \in l, \\
\tilde{B}_{i}\left(x_{1}, x_{2}\right)=\left(\begin{array}{c}
\tilde{B}_{i}^{2}\left(x_{1}, x_{2}\right) \\
\tilde{B}_{i}^{2}\left(x_{2}\right)
\end{array}\right), \quad i \in m l
\end{gathered}
$$

where the first component $\tilde{A}^{1}$, respectively, $\tilde{B_{i}^{1}}$ corresponds to the $\partial / \partial x_{1}$-part of the vector field $\tilde{A}$, respectively, $\tilde{B}_{i}$. On $0\left(x_{0}\right)$ we can define the projection $\pi: 0(x) \rightarrow 0(x) \bmod V^{*}$ by $\pi\left(x_{1}, x_{2}\right)=x_{2}$, see also [8], [9] for a thorough explanation of this "factoring out"-procedure in connection with controlled invariance. For our control system this projection amounts to a quotient system on $0\left(x_{0}\right) \bmod V^{*}$ given by

$$
\dot{x}_{2}=\tilde{A}^{2}\left(x_{2}\right)+\sum_{i=1+1}^{m} \tilde{B}_{i}^{2}\left(x_{2}\right) \tilde{u}_{i}
$$

Because $V^{*} \subset D_{i}^{*}, i \in \boldsymbol{k}$, the distributions $\pi_{*}\left(D_{i}^{*}\right)$ are well defined on $0\left(x_{0}\right)$ mod $V^{*}$ and each of them is involutive (see, e.g., [34]). Setting $\tilde{D}_{i}^{*}=\pi_{*}\left(D_{i}^{*}\right), i \in \boldsymbol{k}$, and $\tilde{\Delta_{0}}=\operatorname{span}\left\{\tilde{B}_{l+1}, \cdots\right.$, $\left.\widetilde{B}_{m}\right\}$ we see by Lemma 4.3 that (3.7) implies

$$
\tilde{\Delta}_{0}=\sum_{i \in k} \tilde{\Delta}_{0} \cap \tilde{D}_{i}^{*}
$$

Moreover, the supremal controlled invariant distribution of (4.14) contained in $\operatorname{Ker} C_{*}$, respectively, $\bigcap_{j \neq i} \operatorname{Ker} C_{j *}, i \in k$, equals $\pi_{*}\left(V^{*}\right)=0$, respectively, $\pi_{*}\left(D_{i}^{*}\right)=\tilde{D}_{i}^{*}, i \in k$. Therefore, we may apply Lemma 4.4 to conclude that (4.7) holds. So by Theorem 4.2 there exists a feedback

$$
\tilde{u}^{2}=\tilde{\alpha}\left(x_{2}\right)+\tilde{\beta}\left(x_{2}\right) v^{2}
$$

(where $\tilde{u}^{2}=\left(\tilde{u}_{l+1}, \cdots, \tilde{u}_{m}\right)^{t}$ ) for the system (4.14) which solves the static-state feedback noninteracting control problem for this system. Getting

$$
v_{i}=\tilde{u}_{i}, \quad i=1, \cdots, l
$$

(4.11), (4.16), and (4.17) together locally define a state feedback which solves the noninteracting control problem for the original system. To show that (3.7) is necessary, let $\left\{R_{i}\right\}_{i \in k}$ be a set of regular local controllability distributions that gives a solution of the decoupling problem, see (4.3)-(4.5) (cf. [24]). Since

$$
\Delta_{0} \subset \sum_{i \in k} \Delta_{0} \cap R_{i} \subset \sum_{i \in k} \Delta_{0} \cap R_{i}^{*}
$$

we see immediately that (3.7) must hold.

Remark: The proof given here is completely different from the corresponding "linear proof" of [15]. In fact, after the tedious calculations of Section III, our proof becomes in the linear case much simpler than in [15].

\section{CONCLUSIONS}

We have proposed a definition of "zeros at infinity" for affine nonlinear control systems, and we demonstrated the usefulness of our definition in the solution of the general decoupling problem. It seems that we have here a promising area of further research. For instance, we expect that the problem of (left and right) invertibility [6], [7], [19], [32] can be studied profitably using the concepts of this paper (see also [23]). Further study can be made of the algebraic aspects of the decoupling problem [21], and of canonical forms in the context [24]. The nonregular input-output decoupling problem remains open to further investigation. An important issue is the existence of global solutions to the decoupling problem; in this connection, we mention the recent work of Byrnes on global controlled invariance. Finally, several aspects of the $V^{*}$-algorithm (2.12) need to be investigated further: among these are the computational side of the algorithm and the study of the consequences of nonconstant dimensions of the distributions $V^{k}$.

\section{REFERENCES}

[1] J. Descusse, J. F. Lafay, and M. Malabre, "On the structure at infinity of linear block-decouplable systems: The general case,"' IEEE Trans. Automat. Contr., vol. AC-28, pp. 1115-1118, 1983.

[2] J. M. Dion, "Feedback block decoupling and infinite structure of linear systems," Int. J. Contr., vol. 37, pp. 521-533, 1983

[3] M. L. J. Hautus, "The formal Laplace transform for smooth linear systems,"' in Mathematical Systems Theory, G. Marchesini and S. K. Mitter, Eds. New York: Springer, 1976 (in Proc. Int. Symp., Udine, Italy, 1975, Lecture Notes in Economics and Mathematical Systems, vol. 131, pp. 29-47).

[4] R. M. Hirschorn, "(A, B)-invariant distributions and disturbance decoupling of nonlinear systems," SIAM J. Contr. Optimiz., vol. 19 , pp. $1-19,1981$.

[5] - "Invertibility of nonlinear control systems," SIAM J. Contr. Optimiz., vol. 17, pp. 289-297, 1979.

[6] - "Invertibility of multivariable nonlinear control systems," IEEE Trans. Automat. Contr., vol. AC-24, pp. 855-865, 1979.

[7] - "Output tracking in multivariable nonlinear systems," IEEE Trans. Automat. Contr., vol. AC-26, pp. 593-595, 1981.

[8] A. Isidori, "The matching of a prescribed linear input-output behavior in a nonlinear system," preprint, 1983; also in IEEE Trans. Automat. Contr., to be published.

[9] A. Isidori, A. J. Krener, C. Gori-Giorgi, and S. Monaco, "Nonlinear dedoupling via feedback: A differential-geometric approach," IEEE Trans. Automat. Contr., vol. AC-26, pp. 331-345, 1981.

[10] - "Locally (f, g)-invariant distributions,"' Syst. Contr. Lett., vol. 1 , pp. $12-15,1981$.

[11] A. Isidori, "Sur la théorie structurelle et le problème du decouplement des perturbations dans les systèmes nonlinéaries," in Outils et Modèles Mathématiques pour l'Automatique, l'Analyse des Systemes et le Traitement du Signal, I.D. Landau, Ed. Paris, France, C.N.R.S., 1981

[12] A. J. Krener and A. Isidori, “(adf, G)-invariant and controllability distributions," in Feedback Control of Linear and Nonlinear Systems D. Hinrichsen and A. Isidori, Eds. (Lecture Notes in Control and Information Sciences, vol. 39, pp. 157-164, 1982). 
[13] M. Malabre, "Structure à l'infini des triplets invariants. Application à la poursuite parfaite de modèle," in Analysis and Optimization of Systems, A. Bensoussan and J. L. Lions, Eds. New York: Springer, 1982, pp. 43-53 (Lecture Notes in Control and Information Sciences, vol. 44).

[14] A.S. Morse, "System invariants under feedback and cascade control," in Mathematical Systems Theory, G. Marchesini and S. K. Mitter, Eds. New York: Springer, 1976, pp. 61-74 (in Proc. Int. Symp., Udine, Italy, 1975, Lecture Notes in Economics and Mathematical Systems, vol. 131).

[15] A. S. Morse and W. M. Wonham, "Status of noninteracting control," IEEE Trans. Automat. Contr., vol. AC-16, pp. 568-581, 1971.

[16] H. Nijmeijer, "Controlled invariance for affine control systems,"' Int. J. Contr., vol. 34, pp. 824-833, 1981.

[17] - "Observability of a class of nonlinear systems: A geometric approach," Ricerche di Automatica, vol. 12, pp. 1-9, 1981.

[18] , "Controllability distributions for nonlinear systems," Syst. Contr. Lett., vol. 2, pp. 122-129, 1982.

[19] _- "Invertibility of affine nonlinear control systems: A geometric approach," Syst. Contr. Lett., vol. 2, pp. 163-168, 1982.

[20] , "Feedback decomposition of nonlinear control systems," IEEE Trans. Automat. Contr., vol. AC-28, pp. 861-862, 1983.

[21] "Noninteracting control for nonlinear systems," in Proc. 22nd $C D C$, San Antonio, TX, 1983.

[22] _ "The triangular decoupling problem for nonlinear control systems,"' Nonlinear Anal. Appl., vol. 8, pp. 273-279, 1984.

[23] , "Zeros at infinity for nonlinear systems, what are they and what are they good for," Dep. Appl. Math., Tech. Univ. Twente, Twente, The Netherlands, Memo. 482.

[24] H. Nijmeijer and J. M. Schumacher, "The noninteracting control problem for nonlinear control system," Dep. Appl. Math., Tech. Univ. Twente, Twente, The Netherlands (revised version, 1984; submitted for publication).

[25] _ "Input-output decoupling of nonlinear systems with an application to robotics," in Analysis and Optimization of Systems, A. Bensoussan and J. L. Lions, Eds. Proc. 6th Int. Conf. Anal. Opt. Syst., Nice, France, June 19-22, 1984, pp. 391-411 (Lecture Notes in Control Information Sciences pt. 2).

[26] H. H. Rosenbrock, "On the inherent integration structure of multivariable nonlinear systems," submitted for publication.

[27] H. Nijmeijer and A. J. van der Schaft, "Controlled invariance for nonlinear systems," IEEE Trans. Automat. Contr., vol. AC-27, pp. 904-914, 1982.

[28] - "The disturbance decoupling problem for nonlinear control systems," IEEE Trans. Automat. Contr., vol. AC-28, pp. 621-623, 1983.

[29] W. Respondek, "On decomposition of nonlinear control systems," Syst. Contr. Lett., vol. 1, pp. 301-308, 1982.

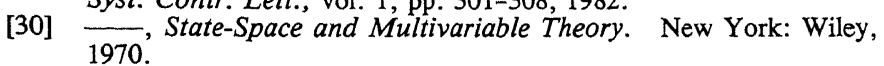

[31] S. N. Singh, "Nonlinear state-variable-feedback excitation and governor control design using decoupling theory," in IEE Proc., Pt. D., vol. 127,1980 , pp. 131-141.

[32] "A modified algorithm for invertibility in nonlinear systems," IEEE Trans. Automat. Contr., vol. AC-26, pp. 595-598, 1981.
[33] H. J. Sussmann and V. Jurdjevic, "Controllability of nonlinear systems," J. Differential Equations, vol. 12, pp. 95-116, 1972.

[34] A. J. van der Schaft, "Observability and controllability for smooth nonlinear systems,"' SIAM J. Contr. Optimiz., vol. 20, pp. 338-354, 1982.

[35] A. I. G. Vardulakis, "On infinite zeros," Int. J. Contr., vol. 32, pp. 849-866, 1980.

[36] G. C. Verghese and T. Kailath, "Rational matrix structure," IEEE Trans. Automat. Contr., vol. AC-26, pp. 434-439, 1981.

[37] W. M. Wonham, Linear Multivariable Control: A.Geometric Approach. New York: Springer, 1979.

[38] W. M. Wonham and A. S. Morse, "Decoupling and pole assignment in linear multivariable systems: A geometric approach," SIAM J. Contr. Optimiz., vol. 9, pp. 1-18, 1970.

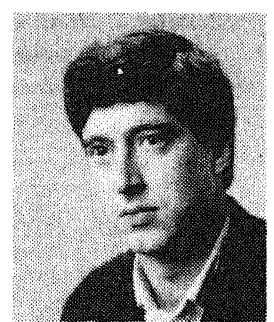

Henk Nijmeijer (M'83) was born in Assen, The Netherlands, on March 16, 1955. He received the "drs." degree in pure mathematics in 1979 and the Ph.D. degree in 1983, both from the University of Groningen, Groningen, The Netherlands.

From January 1980 until March 1983, he was with the Centre for Mathematics and Computer Science, Amsterdam, The Netherlands. Since then he has been with the Department of Applied Mathematics, Technical University of Twente, Enschede. His present research interests are in geometric control theory and dynamical systems.

Dr. Nijmeijer is a member of the Dutch Nonlinear Systems Group.

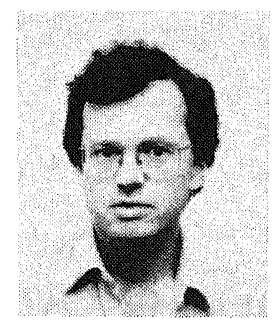

Johannes M. (Hans) Schumacher (M'80) received his training as an applied mathematician at the Vrije Universiteit, Amsterdam, The Netherlands, where he received the "cand." degree in 1972, the "drs." degree in 1976, and the Ph.D. degree in 1981 .

After short-term affilations with the Laboratory for Information and Decision Systems, Massachusetts Institute of Technology, Cambridge, the Econometric Institute, Erasmus University, Rotterdam, and the European Space Technology Centre ESTEC, Noordwijk, The Netherlands, he joined the Centre for Mathematics and Computer Science, Amsterdam, The Netherlands, where is currently a Senior Research Scientist. His present research interests are in the areas of linear and nonlinear systems, and in applications to the theory of mechanical structures.

Dr. Schumacher is a candidate member of the Dutch Nonlinear Systems Group. 